\title{
Selected aspects of quality of life and household cost of care among patients with genital warts attending selected STD clinics in the Western Province of Sri Lanka
}

\author{
Weerasinghe D.P.K., ${ }^{1}$ Rajapaksa L.I. ${ }^{2}$
}

\begin{abstract}
Introduction: Genital warts being a common STI in the country, its impact on quality of life, and cost of care associated with it need to be studied within the current STI context in Sri Lanka.

Objective: To assess selected aspects of disease specific quality of life and its associated factors, response to treatment and household cost of care in-patient with genital warts attending selected STD clinics in the Western Province of Sri Lanka.

Method: Study consisted of descriptive study of 240 patients with genital warts and a longitudinal follow up component to assess response to treatment, disease specific quality of life and household costing associated with genital warts by an interviewer administered questionnaire. Disease specific quality of life among patient with genital warts was assessed with a questionnaire validated by an expert panel. It consisted of 21 items that assess physical, emotional and sexual dimensions separately.
\end{abstract}

Result: Study sample consisted of $151(62.9 \%)$ males and most $(93.8 \%, n=225)$ of participants were Sinhalese. Majority of participants $(79.9 \%, n=192)$ were from age group between 20 to 39 years. More of females $(78.7 \%, n=70)$ in the sample were married than males $(46.4 \%, n=70)$. Cauliflower type of genital warts were the most common type $(56.2 \%, n=135)$ and prepuce and labia majora were the commonly involved sites. There was no statistically significant difference in response to treatment among different treatment. It showed that physical discomfort caused by genital warts and treatment in subsequent visits had not affected their daily activities. However, some of the items in emotional and sexual dimension were affected significantly to most of the participants. Being a male, unmarried, and an idea of having children in future showed statistically significant lower median scores for emotional dimension. They showed similar statistically significant lower scores for sexual dimension except for being unmarried. Median spending for a clinic day among males was Rs 175 and among females was Rs 441.56. Median total time spent by males for complete cure was 35 hours with IC range of 21 - 49 hours compared to 35 hours with IC range of 21 to 56 hours among females.

Conclusion: Patients with genital warts showed significant impairment of disease specific quality of life in relation to emotional and sexual dimensions. It was clear that treatment of genital warts is associated with considerable time loss and expenditure to patients.

Key words: Genital warts, Disease specific quality of life, Costing of care

Authors: corresponding author; ${ }^{1}$ Dr D.P.K.Weerasinghe, MBBS, PgDipVen, DFSRH (UK), MD; Consultant Venereologist, STD Clinic, Chilaw .Email; weerasinghepriyanthak@gmail.com, (iD https://orcid.org/0000-0002-1129-9963

${ }^{2}$ Dr L.I.Ragapaksa, MBBS, MSC, MD, Dip STD Consultant Venereologist, National STD/AIDS Control Programme, Sri Lanka, Email: lilanirajapaksa2@gmail.com, (iD https://orcid.org/0000-0003-3547-1104

Acknowledgement: All Consultant Venereologists in National STD / AIDS Control Programme, Staff in Central STD Clinic, STD Clinic Ragama and Kalubowila, Conflict of interest: No conflict of interest, Funding: No funding support for this study Originality: This is an original work not published anywhere, Submitted: 05.11.2017, Accepted: 15.12.2017 


\section{Full article}

Introduction

Genital wart is the second most commonly reported STI in Sri Lanka.(1) Although genital warts are commonly perceived as a nonserious condition, treatment duration is often long with varying effectiveness. The gravity of patient's suffering, and the impact on quality of life, household cost associated with care are important issues that have not yet been assessed systematically in Sri Lanka. These issues have been discussed extensively in developed countries with the availability of prophylactic quadrivalent vaccine for HPV infection that can prevent $90 \%$ of genital warts (2).

\section{Method}

Study consisted of two phases. Descriptive cross sectional study was used to describe socio-demographic factors, behavioural and clinical characteristics, and baseline disease specific quality of life in 240 patients with genital warts attending central STD clinicColombo, STD clinic-Ragama, STD clinicKalubowila over a period of seven months. All consecutive patients were enrolled if they consented for the study. Further, prospective longitudinal follow up study was used to describe response to treatment and disease specific quality of life in different intervals following treatment and household cost of care for recruited patient with genital warts. Disease specific quality of life was measured with a tool (First version of the Cuestionario Especifico en Condilomas Acuminados CECA used in Europe (3)) validated by expert panel for face and content validity, and it was used to measure the physical, emotional and sexual dimensions among patient with genital warts. It assess the impact during last one week with a rated scale. In assessing disease specific quality of life better score indicates better quality of life. Results were analyzed using SPSS version 16 and when analyzing associated factors for disease specific quality of life, non parametric tests were applied.
Results

Study sample consisted of $151(62.9 \%)$ males and 89 (37.1\%) females and most (93.8\%, $\mathrm{n}=225$ ) of participants were Sinhalese. Majority of participants $(79.9 \%, n=192)$ were from age group between 20 to 39 years. Higher number of females $(58.4 \%, n=52)$ were unemployed with no self income compared to $7 \% \quad(n=10)$ of males. Important occupations identified among males were Tri forces and drivers including three wheeler drivers. (Table 1)

\begin{tabular}{|c|c|c|c|}
\hline Variables & Level & Male & Female \\
\hline \multirow{3}{*}{$\begin{array}{l}\text { Mean Age } \\
\text { SD } \\
\text { Range }\end{array}$} & yrs & 31.50 & 32.03 \\
\hline & & 9.3 & 9.5 \\
\hline & & $18-63$ & $16-49$ \\
\hline \multirow{3}{*}{$\begin{array}{l}\text { Marital } \\
\text { status }\end{array}$} & & $70(46.4 \%)$ & $70(78.7 \%)$ \\
\hline & Unmarried & $79(52.3 \%)$ & 8 (9.0\%) \\
\hline & Other* & $2(1.4 \%)$ & $11(13 \%)$ \\
\hline \multirow{6}{*}{$\begin{array}{l}\text { Employment } \\
\text { status }\end{array}$} & Unemployed & $10(7.0 \%)$ & $52(58.4 \%)$ \\
\hline & Drivers & $16(10.6 \%)$ & 0 \\
\hline & Tri-forces & $31(20.5 \%)$ & $1(1.1 \%)$ \\
\hline & Professional & $15(9.9 \%)$ & $1(1.1 \%)$ \\
\hline & Business & $16(10.5 \%)$ & $20(8.3 \%)$ \\
\hline & Others & $63(41.7 \%)$ & $15(16.85)$ \\
\hline
\end{tabular}

*other -separated/divorced/widow

\section{Behavioural \& Clinical Characteristics}

Behavioural and clinical characteristics of the sample are as follows (Table 2)

\begin{tabular}{|c|c|c|c|}
\hline Variables & Level & Male & Female \\
\hline \multirow{4}{*}{$\begin{array}{l}\text { Sexual } \\
\text { orientation }\end{array}$} & Heterosexual & $110(72.8 \%)$ & $89(100 \%)$ \\
\hline & Same sex & $15(9.9 \%)$ & - \\
\hline & Bisexual & $26(17.2 \%)$ & - \\
\hline & Total & 151 & 89 \\
\hline \multirow{6}{*}{$\begin{array}{l}\text { First Sexual } \\
\text { Exposure }\end{array}$} & Female NRP & $93(61.6 \%)$ & $N A$ \\
\hline & Male NRP & $23(15.2 \%)$ & $15(16.9 \%)$ \\
\hline & FSW & $11(7.3 \%)$ & $N A$ \\
\hline & $M P$ & 24 (15.9\%) & $73(82.0 \%)$ \\
\hline & Client & & $1(1.1 \%)$ \\
\hline & Total & 151 & 89 \\
\hline \multirow{3}{*}{$\begin{array}{l}\text { Lifetime } \\
\text { sexual } \\
\text { partners }\end{array}$} & 1 & $18(11.9 \%)$ & 64 (71.9\%) \\
\hline & $\geq 1$ & $133(88.1 \%)$ & $25(28.1 \%)$ \\
\hline & Total & 151 & 89 \\
\hline \multirow{4}{*}{$\begin{array}{l}\text { Morphological } \\
\text { type }\end{array}$} & Cauliflower & $85(56.3 \%)$ & $50(56.2 \%)$ \\
\hline & Papular & 24 (15.9\%) & $7(7.9 \%)$ \\
\hline & Flat & $5 \quad(3.3 \%)$ & $2(2.2 \%)$ \\
\hline & > one type & $37(24.5 \%)$ & $30(33.7 \%)$ \\
\hline \multirow{5}{*}{$\begin{array}{l}\text { Area Involved } \\
*\end{array}$} & Prepuce & $65(43.0 \%)$ & $N A$ \\
\hline & Labia Majora & $N A$ & $51(57.3 \%)$ \\
\hline & Penile shaft & $40(26.2 \%)$ & \\
\hline & Labiaminora & $N A$ & $37(41.6 \%)$ \\
\hline & Coronal sulcus & $22(14.2 \%)$ & \\
\hline
\end{tabular}

\section{Response to Treatment}


Almost $68 \%(n=102)$ of males and $53 \%(n=47)$ females got their genital warts cleared by three months. Most common mode of treatment was trichoroacetic acid (TCA) in both males and females followed by Liquid Nitrogen. Usage of Imiquimod was very low. Out of 240 patients final treatment outcome was available for 166 (69.2\%) patients.(table 3)

Table 3: Distribution of outcome among participants based on treatment used

\begin{tabular}{|l|r|r|r|}
\hline \multirow{2}{*}{$\begin{array}{l}\text { Treatment } \\
\text { Used }\end{array}$} & \multicolumn{3}{|c|}{ Curnal Outcome } \\
\cline { 2 - 4 } & $76(92.7 \%)$ & No Cure & Total \\
\hline TCA & $29(87.3 \%)$ & $82(100 \%)$ \\
\hline Liquid Nitrogen & $4(12.1 \%)$ & $33(100 \%)$ \\
\hline Combinations & $41(80.4 \%)$ & $10(19.6 \%)$ & $51(100 \%)$ \\
\hline Total & 146 & 20 & 166 \\
\hline \multicolumn{4}{|l|}{ TCA-trichloroacetic acid } \\
\hline
\end{tabular}

There was no statistically significant difference in response to treatment among different treatment groups $(p=0.106)$

\section{Disease Specific Quality of Life}

It showed that physical discomfort caused by genital warts and treatment in subsequent visits had not affected their daily activities. However, some of the items in emotional and sexual dimension were affected significantly to most of the participants. Table 4 describes baseline figures in Disease specific quality of life.

In one week, severity of emotional worry had decreased and this is more obvious at 1 month follow up.(Table $5 \& 6$ )

Table 4: Percentage of study population rated each response in the items of the Disease Specific QOL dimensions at baseline

\begin{tabular}{|c|c|c|c|c|c|c|c|}
\hline \multirow{2}{*}{\multicolumn{2}{|c|}{$\begin{array}{l}\text { Description of physical, emotional and } \\
\text { sexual dimension }\end{array}$}} & \multicolumn{5}{|c|}{ Response } & \multirow[b]{2}{*}{ Total } \\
\hline & & \multirow{2}{*}{$\begin{array}{r}\text { Always } \\
(1) \\
0(0 \%)\end{array}$} & \multirow{2}{*}{\begin{tabular}{r|} 
Almost \\
Always (2) \\
$0(0 \%)$
\end{tabular}} & \multirow{2}{*}{\begin{tabular}{r|}
$\begin{array}{r}\text { Sometimes } \\
(3)\end{array}$ \\
$1(0.4 \%)$ \\
\end{tabular}} & \multirow{2}{*}{\begin{tabular}{|r|} 
Rarely \\
$(4)$ \\
$28(11.7 \%)$ \\
\end{tabular}} & \multirow{2}{*}{$\begin{array}{r}\text { Never } \\
(5) \\
211(87.9 \%)\end{array}$} & \\
\hline P1 & Discomfort & & & & & & $240(100 \%)$ \\
\hline $\mathrm{P} 2$ & Pain following treatment & $0(0 \%)$ & $0(0 \%)$ & $0(0 \%)$ & $0(0 \%)$ & $240(100 \%)$ & $240(100 \%)$ \\
\hline E 1 & Personal Hygiene & $35(14.6 \%)$ & $72(30.0 \%)$ & $60(25.0 \%)$ & $12(5.0) \%$ & $61(25.4 \%)$ & $240(100 \%)$ \\
\hline E 2 & Want disappear & $61(25.4 \%)$ & $147(61.3 \%)$ & $22(9.2 \%)$ & $5(2.1 \%)$ & $5(2.1 \%)$ & $240(100 \%)$ \\
\hline E 3 & Chronic infection & $53(22.1 \%)$ & $135(56.3 \%)$ & $33(13.8 \%)$ & $8(3.3 \%)$ & $11(4.6 \%)$ & $240(100 \%)$ \\
\hline E 4 & Will get worse & $67(27.9 \%)$ & $133(55.4 \%)$ & $21(8.8 \%)$ & $8(3.3 \%)$ & $11(4.6 \%)$ & $240(100 \%)$ \\
\hline E 5 & Feeling of guilt & $70(29.2 \%)$ & $63(26.3 \%)$ & $12(5.0 \%)$ & $3(1.3 \%)$ & $92(38.3 \%)$ & $240(100 \%)$ \\
\hline E 6 & Who infect me & $41(17.1 \%)$ & $105(43.8 \%)$ & $18(7.5 \%)$ & $9(3.8 \%)$ & $67(27.9 \%)$ & $240(100 \%)$ \\
\hline E 7 & Infect my partner & $84(35.0 \%)$ & $85(35.4 \%)$ & $13(5.4 \%)$ & $5(2.1 \%)$ & $53(22.1 \%)$ & $240(100 \%)$ \\
\hline E 8 & Mind anxious & $52(21.7 \%)$ & $106(44.2 \%)$ & $60(25.0 \%)$ & $11(4.6 \%)$ & $11(4.6 \%)$ & $240(100 \%)$ \\
\hline E 9 & Felt insecure & $32(13.3 \%)$ & $74(30.8 \%)$ & $55(22.9 \%)$ & $16(6.7 \%)$ & $63(26.3 \%)$ & $240(100 \%)$ \\
\hline E10 & Stress affected daily activities & $16(6.7 \%)$ & $35(14.6 \%)$ & $21(8.8 \%)$ & $26(10.8 \%)$ & $142(59.2 \%)$ & $240(100 \%)$ \\
\hline E11 & Problems in having children & $30(12.5 \%)$ & $61(25.8 \%)$ & $21(8.8 \%)$ & $14(5.8 \%)$ & $114(47.5 \%)$ & $240(100 \%)$ \\
\hline E12 & Lacking treatment knowledge & $15(6.3 \%)$ & $89(37.1 \%)$ & $34(14.2 \%)$ & $15(6.3 \%)$ & $87(36.3 \%)$ & $240(100 \%)$ \\
\hline E13 & Knowing other about GW & $44(18.3 \%)$ & $135(56.3 \%)$ & $23(9.6 \%)$ & $14(5.8 \%)$ & $24(10.0 \%)$ & $240(100 \%)$ \\
\hline E14 & Treatment for long time & $27(11.3 \%)$ & $90(37.5 \%)$ & $29(12.1 \%)$ & $13(5.4 \%)$ & $81(33.8 \%)$ & $240(100 \%)$ \\
\hline E15 & $\begin{array}{l}\text { Social relationship with partner } \\
\text { affected }\end{array}$ & $2(0.8 \%)$ & $13(5.4 \%)$ & $8(3.3 \%)$ & $20(8.3 \%)$ & $197(82.1 \%)$ & $240(100 \%)$ \\
\hline S1 & Sexual drive decreased & 19(7.9\%) & $79(32.9 \%)$ & $37(15.4 \%)$ & $11(4.6 \%)$ & $95(39.2 \%)$ & $240(100 \%)$ \\
\hline $\mathrm{S} 2$ & Avoid sexual relations & $84(35.0 \%)$ & $64(26.6 \%)$ & $38915.8 \%)$ & $5(2.1 \%)$ & $49(20.4 \%)$ & $240(100 \%)$ \\
\hline S3 & Worried during sex* & $8(11.1 \%)$ & $19(26.4 \%)$ & $22(30.6 \%)$ & $4(5.6 \%)$ & $19(26.4 \%)$ & $72(100 \%)$ \\
\hline S4 & Sexual relations decreased* & $7(9.7 \%)$ & $31(43.1 \%)$ & $16(22.2 \%)$ & $3(4.2 \%)$ & $15(20.8 \%)$ & $72(100 \%)$ \\
\hline S5 & Worried to have condoms+ & $1(7.1 \%)$ & $2(14.3 \%)$ & $7(50.0 \%)$ & $2(14.3 \%)$ & $2(14.3 \%)$ & $14(100 \%)$ \\
\hline
\end{tabular}


Table 5 : Percentage of study population rated each response in the items of the Disease Specific $\mathrm{QOL}$ dimensions at one week

\begin{tabular}{|c|c|c|c|c|c|c|c|}
\hline \multirow{2}{*}{\multicolumn{2}{|c|}{$\begin{array}{l}\text { Description of physical, emotional and } \\
\text { sexual dimension }\end{array}$}} & \multicolumn{5}{|c|}{ Response } & \multirow{3}{*}{$\begin{array}{r}\text { Total } \\
211(100 \%)\end{array}$} \\
\hline & & \multirow{2}{*}{$\begin{array}{r}\text { Always } \\
(1) \\
0(0 \%)\end{array}$} & \multirow{2}{*}{\begin{tabular}{|r|} 
Almost Always \\
$(2)$ \\
$0(0 \%)$
\end{tabular}} & \multirow{2}{*}{\begin{tabular}{r|} 
Sometimes \\
$(3)$ \\
$4(1.9 \%)$
\end{tabular}} & \multirow{2}{*}{\begin{tabular}{r|}
$\begin{array}{r}\text { Rarely } \\
(4)\end{array}$ \\
$16(7.6 \%)$
\end{tabular}} & \multirow{2}{*}{$\begin{array}{r}\begin{array}{r}\text { Never } \\
(5)\end{array} \\
191(90.5 \%)\end{array}$} & \\
\hline P1 & Discomfort & & & & & & \\
\hline P2 & Pain following treatment & $1(0.5 \%)$ & $0(0 \%)$ & $13(6.2 \%)$ & $37(17.5 \%)$ & $160(75.8 \%)$ & $211(100 \%)$ \\
\hline E 1 & Personal Hygiene & $10(4.7 \%)$ & $32(15.2 \%)$ & $71(33.6 \%)$ & $47(22.3 \%)$ & $51(24.2 \%)$ & $211(100 \%)$ \\
\hline E 2 & Want disappear & $14(6.6 \%)$ & $61(28.9 \%)$ & $52(24.6 \%)$ & $54(25.6 \%)$ & $30(14.2 \%)$ & $211(100 \%)$ \\
\hline E 3 & Chronic infection & $19(9.0 \%)$ & $118(55.9 \%)$ & $55(26.1 \%)$ & $12(5.7 \%)$ & $7(3.3 \%)$ & $211(100 \%)$ \\
\hline E 4 & Will get worse & $13(6.2 \%)$ & $60(28.4 \%)$ & $40(19.0 \%)$ & $69(32.7 \%)$ & $29(13.7 \%)$ & $211(100 \%)$ \\
\hline E 5 & Feeling of guilt & $35(16.6 \%)$ & $79(37.4 \%)$ & $17(8.1 \%)$ & $10(4.7 \%)$ & $70(33.2 \%)$ & $211(100 \%)$ \\
\hline E 6 & Who infect me & $21(10.0 \%)$ & $97(46.0 \%)$ & $28(13.3 \%)$ & $13(6.2 \%)$ & $52(24.6 \%)$ & $211(100 \%)$ \\
\hline E 7 & Infect my partner & $41(19.4 \%)$ & $85(40.3 \%)$ & $33(15.6 \%)$ & $8(3.8 \%)$ & $44(20.9 \%)$ & $211(100 \%)$ \\
\hline E 8 & Mind anxious & $12(5.7 \%)$ & $41(19.4 \%)$ & $72(34.1 \%)$ & $55(26.1 \%)$ & $31(14.7 \%)$ & $211(100 \%)$ \\
\hline E 9 & Felt insecure & $9(4.3 \%)$ & $27(12.8 \%)$ & $50(23.7 \%)$ & $36(17.1 \%)$ & $89(42.2 \%)$ & $211(100 \%)$ \\
\hline E10 & Stress affected daily activities & $2(0.9 \%)$ & $11(5.2 \%)$ & $26(12.3 \%)$ & $34(16.1 \%)$ & $138(65.4 \%)$ & $211(100 \%)$ \\
\hline E11 & Problems in having children & $7(3.3 \%)$ & $33(15.6 \%)$ & $41(19.4 \%)$ & $24(11.4 \%)$ & $106(50.2 \%)$ & $211(100 \%)$ \\
\hline E12 & Lacking treatment knowledge & $4(1.9 \%)$ & $25(11.8 \%)$ & $29(13.7 \%)$ & $37(17.5 \%)$ & $116(55.0 \%)$ & $211(100 \%)$ \\
\hline E13 & Knowing other about GW & $15(7.1 \%)$ & $125(59.5 \%)$ & $35(16.7 \%)$ & $15(7.1 \%)$ & $20(9.5 \%)$ & $211(100 \%)$ \\
\hline E14 & Treatment for long time & $12(5.7 \%)$ & $54(25.6 \%)$ & $39(18.5 \%)$ & $25(11.8 \%)$ & $81(38.4 \%)$ & $211(100 \%)$ \\
\hline E15 & $\begin{array}{l}\text { Social relationship with partner } \\
\text { affected }\end{array}$ & & $5(2.4 \%)$ & $6(2.8 \%)$ & $20(9.5 \%)$ & $180(85.3 \%)$ & $211(100 \%)$ \\
\hline S1 & Sexual drive decreased & $10(4.7 \%)$ & $61(25.6 \%)$ & $31(14.7 \%)$ & $18(8.5 \%)$ & $91(43.1 \%)$ & $211(100 \%)$ \\
\hline S2 & Avoid sexual relations & $111(52.6 \%)$ & $40(19.0 \%)$ & $21(10.0 \%)$ & $7(3.3 \%)$ & $32(15.2 \%)$ & $211(100 \%)$ \\
\hline S3 & Worried during sex* & $3(9.7 \%)$ & $9(29.0 \%)$ & $12(38.7 \%)$ & $3(9.7 \%)$ & $4(12.9 \%)$ & $31(100 \%)$ \\
\hline S4 & Sexual relations decreased* & $2(6.5 \%)$ & $15(48.4 \%)$ & $8(25.8 \%)$ & $3(9.7 \%)$ & $3(9.7 \%)$ & $31(100 \%)$ \\
\hline S5 & Worried to have condoms+ & $\begin{array}{c}-1(9.0 \%) \\
1(9.0 \%)\end{array}$ & $6(54.5 \%)$ & $1(9.0 \%)$ & $0(0 \%)$ & $3(27.3 \%)$ & $11(100 \%)$ \\
\hline
\end{tabular}

$P=$ Physical dimension, E-Emotional dimension, S-Sexual dimension

For items of sexual dimension responses were Totally agree (1), Almost agree (2), Agree to a certain extent (3), Difficult to agree (4), Not agree at all (5) * * Item applied only for 31 participants and + for 11 participants.

Table 6 : Percentage of study population rated each response in the items of the Disease Specific QOL dimensions at one month

\begin{tabular}{|c|c|c|c|c|c|c|c|}
\hline \multirow{2}{*}{\multicolumn{2}{|c|}{$\begin{array}{l}\text { Description of physical, emotional and } \\
\text { sexual dimension }\end{array}$}} & \multicolumn{5}{|c|}{ Response } & \multirow{3}{*}{$\begin{array}{r}\text { Total } \\
108(100 \%)\end{array}$} \\
\hline & & \multirow{2}{*}{$\begin{array}{r}\text { Always } \\
(1) \\
0(0 \%)\end{array}$} & \multirow{2}{*}{\begin{tabular}{|r|} 
Almost Always \\
$(2)$ \\
$0(0 \%)$ \\
\end{tabular}} & \multirow{2}{*}{\begin{tabular}{r|} 
Sometimes \\
$(3)$ \\
$1(0.9 \%)$
\end{tabular}} & \multirow{2}{*}{\begin{tabular}{r|} 
Rarely \\
$(4)$ \\
$7(6.5 \%)$
\end{tabular}} & \multirow{2}{*}{$\begin{array}{r}\begin{array}{r}\text { Never } \\
(5)\end{array} \\
100(92.6 \%)\end{array}$} & \\
\hline P1 & Discomfort & & & & & & \\
\hline P2 & Pain following treatment & $0(0 \%)$ & $0(0 \%)$ & $4(3.7 \%)$ & $13(12.0 \%)$ & $91(84.3 \%)$ & $108(100 \%)$ \\
\hline E 1 & Personal Hygiene & $6(5.6 \%)$ & $11(10.2 \%)$ & $32(29.6 \%)$ & $19(17.6 \%)$ & $40(37.0 \%)$ & $108(100 \%)$ \\
\hline E 2 & Want disappear & $5(4.6 \%)$ & $16(14.8 \%)$ & $27(25.0 \%)$ & $32(29.6 \%)$ & $28(25.9 \%)$ & $108(100 \%)$ \\
\hline E 3 & Chronic infection & $5(4.6 \%)$ & $59(54.6 \%)$ & $25(23.1 \%)$ & $9(8.3 \%)$ & $10(9.3 \%)$ & $108(100 \%)$ \\
\hline E 4 & Will get worse & $4(3.7 \%)$ & $21(19.4 \%)$ & $21(19.4 \%)$ & $27(25.0 \%)$ & $35(32.4 \%)$ & $108(100 \%)$ \\
\hline E 5 & Feeling of guilt & $9(8.3 \%)$ & $42(38.9 \%)$ & $13(12.0 \%)$ & $3(2.8 \%)$ & $41(38.0 \%)$ & $108(100 \%)$ \\
\hline E 6 & Who infect me & $7(6.5 \%)$ & $34(31.5 \%)$ & $19(17.6 \%)$ & $8(7.4 \%)$ & $40(37.0 \%)$ & $108(100 \%)$ \\
\hline E 7 & Infect my partner & $8(7.4 \%)$ & $46(42.6 \%)$ & $18(16.7 \%)$ & $2(1.9 \%)$ & $34(31.5 \%)$ & $108(100 \%)$ \\
\hline E 8 & Mind anxious & $2(1.9 \%)$ & $13(12.0 \%)$ & $31(28.7 \%)$ & $31(28.7 \%)$ & $31(28.7 \%)$ & $108(100 \%)$ \\
\hline E9 & Felt insecure & $2(1.9 \%)$ & $9(8.3 \%)$ & $14(13.0 \%)$ & $17(15.7 \%)$ & $66(61.1 \%)$ & $108(100 \%)$ \\
\hline E10 & Stress affected daily activities & $0(0 \%)$ & $2(1.9 \%)$ & $6(5.6 \%)$ & $11(10.2 \%)$ & $89(82.4 \%)$ & $108(100 \%)$ \\
\hline E11 & Problems in having children & $1(0.9 \%)$ & $11(10.2 \%)$ & $21(19.4 \%)$ & $7(6.5 \%)$ & $68(63.0 \%)$ & $108(100 \%)$ \\
\hline E12 & Lacking treatment knowledge & $1(0.9 \%)$ & $3(2.8 \%)$ & $10(9.3 \%)$ & $18(16.7 \%)$ & $76(70.4 \%)$ & $108(100 \%)$ \\
\hline E13 & Knowing other about GW & $7(6.5 \%)$ & $57(52.8 \%)$ & $18(16.7 \%)$ & $11(10.2 \%)$ & $15(13.9 \%)$ & $108(100 \%)$ \\
\hline E14 & Treatment for long time & $2(1.9 \%)$ & $21(19.4 \%)$ & $22(20.4 \%)$ & $12(11.1 \%)$ & $51(47.2 \%)$ & $108(100 \%)$ \\
\hline E15 & $\begin{array}{l}\text { Social relationship with partner } \\
\text { affected }\end{array}$ & 0 & $2(1.9 \%)$ & 0 & $3(2.8 \%)$ & $103(95.4 \%)$ & $108(100 \%)$ \\
\hline S1 & Sexual drive decreased & $5(4.6 \%)$ & $23(21.3 \%)$ & $21(19.4 \%)$ & $6(5.6 \%)$ & $53(49.1 \%)$ & $108(100 \%)$ \\
\hline S2 & Avoid sexual relations & $54(50.0 \%)$ & $13(12.0 \%)$ & $14(13.0 \%)$ & $3(2.8 \%)$ & $24(22.2 \%)$ & $108(100 \%)$ \\
\hline S3 & Worried during sex* & $1(4.8 \%)$ & $6(28.6 \%)$ & $8(38.1 \%)$ & $1(4.8 \%)$ & $5(23.8 \%)$ & $21(100 \%)$ \\
\hline S4 & Sexual relations decreased* & $0(0 \%)$ & $9(42.9 \%)$ & $4(19.0 \%)$ & $3(14.3 \%)$ & $5(23.8 \%)$ & $21(100 \%)$ \\
\hline S5 & Worried to have condoms+ & $0(0 \%)$ & $6(50.0 \%)$ & $2(16.7 \%)$ & $2(16.7 \%)$ & $2(16.7 \%)$ & $12(100 \%)$ \\
\hline
\end{tabular}

$P=$ Physical dimension, E-Emotional dimension, S-Sexual dimension

For items of sexual dimension responses were Totally agree (1), Almost agree (2), Agree to a certain extent (3), Difficult to agree (4), Not agree at all (5) * * Item applied only for 21 participants and + for 12 participants. 
Males are more emotionally affected than females ( $p=0.03$ ), those who were unmarried more emotionally affected compared to married ( $p=0.012$ ) and those who had idea of having children in future were more affected compared to those who had no idea of having children $(p=0.001)$ at baseline.

Similarly, males were more affected sexually than females ( $p=0.001)$, and those who had idea of having children in future were sexually affected more compared to those who had no idea of having children $(p=0.017)$ at baseline. Number of genital warts, surface area involved by genital warts have not contributed to statistically significant lower median scores of emotional or sexual dimensions. Similarly educational level, disclosure status, knowledge on genital warts have not contributed for statistically significant lower median scores of emotional or sexual dimensions. (Table 7)

Table 7 : Factors affecting Emotional and Sexual Dimension of Disease Specific Quality of Life

\begin{tabular}{|c|c|c|c|c|c|c|c|c|}
\hline \multirow[t]{2}{*}{ Factor } & \multirow[t]{2}{*}{ Level } & \multirow{2}{*}{$\begin{array}{l}\text { Group } \\
\text { vs. } \\
\text { group }\end{array}$} & \multicolumn{3}{|c|}{$\begin{array}{l}\text { Emotional Dimension of } \\
\text { Disease Specific Quality of Life }\end{array}$} & \multicolumn{3}{|c|}{$\begin{array}{r}\text { Sexual Dimensionof Disease } \\
\text { Specific Quality of Life }\end{array}$} \\
\hline & & & Number & Median & Sig* & Number & Median & Sig* \\
\hline \multirow[t]{6}{*}{ Sex } & \multirow[t]{2}{*}{ Baseline } & Male & 151 & 2.8667 & \multirow{2}{*}{$p=0.03$} & 151 & 2.5000 & \multirow{2}{*}{$p=0.001$} \\
\hline & & Female & 89 & 3.0667 & & 89 & 3.0000 & \\
\hline & \multirow[t]{2}{*}{1 Week } & Male & 143 & 3.4667 & \multirow{2}{*}{$p=0.464$} & 143 & 2.7500 & \multirow{2}{*}{$p=0.087$} \\
\hline & & Female & 69 & 3.4667 & & 69 & 2.7500 & \\
\hline & \multirow[t]{2}{*}{1 Month } & Male & 66 & 3.7333 & \multirow{2}{*}{$p=0.661$} & 66 & 3.0000 & \multirow{2}{*}{$p=0.053$} \\
\hline & & Female & 42 & 3.9000 & & 42 & 3.0000 & \\
\hline \multirow{2}{*}{$\begin{array}{l}\text { Education } \\
\text { level (at baseline) }\end{array}$} & & Grade $\leq 11$ & 142 & 2.9667 & \multirow{2}{*}{$p=0.825$} & 142 & 3.0000 & \multirow{2}{*}{$p=0.185$} \\
\hline & Grade & or above & 98 & 2.9333 & & 98 & 2.5000 & \\
\hline \multirow[t]{2}{*}{ Marital status } & Curre & y married & 140 & 3.000 & \multirow{2}{*}{$p=0.012$} & 140 & 3.0000 & \multirow{2}{*}{$p=0.140$} \\
\hline & Currentl & unmarried & 100 & 2.8667 & & 100 & 2.5000 & \\
\hline \multirow{3}{*}{$\begin{array}{l}\text { Area involved } \\
\text { By Genital warts }\end{array}$} & & $<50 \mathrm{~mm}^{2}$ & 104 & 2.9333 & \multirow{3}{*}{$p=0.296$} & 104 & 3.0000 & \multirow{3}{*}{$p=0.288$} \\
\hline & & $200 \mathrm{~mm}^{2}$ & 101 & 3.0000 & & 101 & 2.5000 & \\
\hline & & $200 \mathrm{~mm}^{2}$ & 34 & 3.1333 & & 34 & 2.5000 & \\
\hline \multirow{4}{*}{$\begin{array}{l}\text { Number of } \\
\text { Genital warts }\end{array}$} & & $1-2$ & 40 & 3.0333 & \multirow{4}{*}{$p=0.296$} & 40 & 3.0000 & \multirow{4}{*}{$p=0.461$} \\
\hline & & $3-5$ & 66 & 2.9000 & & 66 & 3.0000 & \\
\hline & & $6-10$ & 64 & 2.9000 & & 64 & 2.5000 & \\
\hline & & re than 10 & 70 & 3.0333 & & 70 & 2.5000 & \\
\hline \multirow{2}{*}{$\begin{array}{l}\text { Disclosure } \\
\text { status }\end{array}$} & & No & 158 & 2.9333 & \multirow{2}{*}{$p=0.926$} & 158 & 2.5000 & \multirow{2}{*}{$p=0.815$} \\
\hline & & Yes & 82 & 3.0000 & & 82 & 3.0000 & \\
\hline \multirow{2}{*}{$\begin{array}{l}\text { Hope to have } \\
\text { children }\end{array}$} & & Yes & 147 & 2.8667 & \multirow{2}{*}{$p=0.001$} & 147 & 2.5000 & $n-0017$ \\
\hline & & No & 85 & 3.0000 & & 85 & 3.0000 & $p=0.011$ \\
\hline Knowledge on & & Poor & 49 & 3.0000 & $n=027$ & 49 & 2.5000 & $n=0.974$ \\
\hline Genital warts & & Good & 185 & 2.9333 & $p=0.217$ & 185 & 3.0000 & $p=0.9 / 4$ \\
\hline
\end{tabular}

\section{Household Cost}

Median spending for a clinic day among males was 175Rs. (range 0- 3,000Rs) and among females was 441.56Rs. (range 0-1,900). Among those who had complete cure $(60.9 \%$, $\mathrm{n}=84$ ) had episode cost of less than 1000 Rs. and this was seen in both males and females. Nearly $11 \%(n=15)$ had episode cost $>5000$ Rs by the time of cure. Total time spent for cure was available for 146 participants. Seventy three percent $(n=73)$ of males spent less than 42 hours for complete cure whereas nearly $67 \%(n=31)$ of females less than 42 hours for cure. Median total time spent by males for complete cure was 35 hours (IQR=21-49 hours) compared to 35 hours (IQR=21-56 hours) among females. 
Table 8. Distribution of study subject in relation to total cost, episode cost, total time spent per visit and total time spend for cure)

\begin{tabular}{|c|c|c|c|c|c|c|c|}
\hline Variable & Level & Male & $\%$ & Female & $\%$ & Total & $\%$ \\
\hline \multirow{9}{*}{$\begin{array}{l}\text { Total cost in rupees per } \\
\text { visit }\end{array}$} & $\leq 100$ & 52 & 37.1 & 26 & 38.2 & 78 & 37.5 \\
\hline & $101-250$ & 35 & 25.0 & 10 & 14.7 & 45 & 21.6 \\
\hline & $251-500$ & 19 & 13.6 & 10 & 14.7 & 29 & 13.9 \\
\hline & $501-750$ & 9 & 6.4 & 8 & 11.8 & 17 & 8.2 \\
\hline & $751-1000$ & 5 & 3.5 & 4 & 5.9 & 9 & 4.3 \\
\hline & $>1000$ & 20 & 14.3 & 10 & 14.7 & 30 & 14.4 \\
\hline & Total & 140 & - & 68 & - & 208 & 100 \\
\hline & Defaulted & 5 & - & 12 & - & 17 & - \\
\hline & Missing data & 5 & - & 8 & - & 13 & - \\
\hline \multirow{8}{*}{$\begin{array}{l}\text { Episode Cost in rupees } \\
\text { for cure }\end{array}$} & $\leq 1000$ & 60 & 61.8 & 24 & 58.5 & 84 & 60.9 \\
\hline & $1001-2000$ & 16 & 16.5 & 4 & 9.8 & 20 & 14.5 \\
\hline & $2001-3000$ & 5 & 5.2 & 5 & 12.2 & 10 & 7.2 \\
\hline & $3001-4000$ & 4 & 4.1 & 2 & 4.9 & 6 & 4.3 \\
\hline & $4001-5000$ & 2 & 2.1 & 1 & 2.4 & 3 & 2.2 \\
\hline & $>5000$ & 10 & 10.3 & 5 & 12.2 & 15 & 10.9 \\
\hline & Total & 97 & - & 41 & - & 138 & 100 \\
\hline & Missing data & 3 & - & 5 & - & 8 & - \\
\hline \multirow{7}{*}{$\begin{array}{l}\text { Total time spent per visit } \\
\text { in hours }\end{array}$} & $\leq 2$ & 26 & 18.6 & 8 & 11.8 & 34 & 16.3 \\
\hline & $2-4$ & 81 & 57.9 & 34 & 50.0 & 115 & 55.3 \\
\hline & $4-6$ & 19 & 13.5 & 18 & 26.5 & 37 & 17.8 \\
\hline & $6-8$ & 7 & 5.0 & 6 & 8.8 & 13 & 6.2 \\
\hline & $8-10$ & 2 & 1.4 & 1 & 1.5 & 3 & 1.4 \\
\hline & $>10$ & 5 & 3.6 & 1 & 1.5 & 6 & 2.9 \\
\hline & Total & 140 & 100 & 68 & 100 & 208 & 100 \\
\hline
\end{tabular}

\section{Discussion}

The study showed more males with Genital warts compared to females. This has been observed in the STD clinic data over last 10 years in Sri Lanka.(1) Similar distribution was observed in the United Kingdom in patient with first episode of Genital warts.(4) Possibility of higher health seeking behaviour among men could be another reason. However, this study was aclinic based study, and it could not comment on population level data.

Age at first sex among males is decreasing according to the available literature.(6) In the present study mean age of first sex among males was lower (20.56 yrs) than that of females (21.13 yrs). It is interesting to note

that $82 \%$ of females with genital warts had their first sexual contact with marital partner.
However, only $15.9 \%$ of males had first sex with their marital partner. This has been observed in many literature and usually their first contact with a sex worker.(6) In present study $7.3 \%$ of males had first sex with a sex worker while nearly $77 \%$ had first sexual contact with a non regular partner. Number of sexual partners among males were high compared to females. This study showed nearly $72 \%$ of females had only one life time partner while that figure for males was nearly $12 \%$. It reflect that for STI control in Sri Lanka, more interventions need to be focused on males.

Generally the incubation period of genital warts is 3-8 months following sexual contact, but sometimes it could go up to 3 years. $(7,8)$ Out of 89 female 60 had sexual contact only with marital partner during last three years, and it indicates that among females with genital warts most likely source of infection is their marital partner. It is interesting to know that commercial sex workers were not the 
main source of infection for males with genital warts.

Out of available follow up data 146 out of 166 (nearly $88 \%$ ) had complete response within 3 months. Median number of visits for complete cure was 5 . This study findings on response with different treatment options showed no statistically significant superiority of available treatment options and it has been proven in previous studies as well.

Genital warts are generally considered as a mild condition. However, they can cause local irritation, discomfort or bleeding. $(8,9)$ This study showed that physical problems that caused by genital warts do not affect the daily life of patients. Further, even after treatment it showed that physical dimension has not significantly affected day today life.

This study showed that some of the items in the emotional dimension have caused severe problems for more than $50 \%$ of the participants. They have answered those components disturbed their life "Always" or " Almost always". It is clear that following components affected their emotions more than $75 \%$ of the participants as "Always" or " Almost always" at baseline.

Emotional item 2 - I was afraid that the lesions won't disappear

Emotional item 3 - I was anxious to know whether I am going to recover from the virus for good

Emotional item 4 - I worried about whether the warts will get worse

Emotional item13- I worried about people finding out about my illness

However, in one week, severity of emotional worry had decreased and this is more obvious at 1 month follow up. These issues need to be considered in counseling in patients with genital warts.

It is clear that more than $50 \%$ of the participants responded as "Totally agree" or "Almost agree" for the item of avoidance of sexual relations at baseline. Only $30 \%$ of them had sexual contacts during last week and $40 \%$ felt that their sexual drive has decreased. When it comes to one week and one month
$72 \%$ and $62 \%$ of participants had avoided sexual exposures due to genital warts respectively. It is interesting to know that how it change with recovery form genital warts. However, this study would not be able to answer it as once they cleared the lesions they would not attend for follow up.

It was seen that males had statistically significant lower emotional dimension and sexual dimension scores compared to females at base line. This study showed that more females had only one lifetime sexual partner compared to more partners among males. Likelihood of infection from an unfaithful relationship may be the contributing factor for males to have worse emotional and sexual dimension scores compared to females.

However, even in subsequent visits males showed that lower emotional scores compared to females although it was not statistically significant. Ongoing counseling sessions, response to treatment may contribute to improvement of the scores of emotional dimension of QOL.

Current marital status was a significant factor that contribute to lower emotional scores. It was seen that median scores of emotional dimension was lower among "currently unmarried" group compared to currently married group. This difference was statistically significant. In the emotional dimension, doubts of transmission of infection to future partners, transmission of infection to children in the future have contributed for lower scores among those who "currently unmarried".

Further, there was an statistically significant lower median scores in emotional and sexual dimension among those who wish to have children as compared to those who have completed their families at baseline. Doubts on infection transmission to future partners and children have contributed for lower scores among those who wish to have children in the future.

Quadrivalent HPV vaccine would be the ideal choice to expanded programme of immunization (EPI) as it is known to prevent 
$70 \%$ of cervical cancer and $90 \%$ of genital warts. Further, Quadrivalent HPV vaccine could be considered for the future partner as they continued to have concerns on possibility of transmitting the infection to partners. The present study shows that patients with genital warts spend considerable amount of money and time when attending clinics for care. Making availability of patient applied medications would result in saving money and time. Patients with genital warts need to be counseled in detail by medical officers at first and subsequent visits to improve their disease specific quality of life. They need to specifically pay attention in counseling on the risk of transmission of infection to future partners and children, natural history of genital warts, predicted response to treatment

\section{Conclusions}

Patients with genital warts showed significant impairment of disease specific quality of life in relation to emotional and sexual dimensions, and being a male, currently being unmarried, having not completed family life had statistically significant association with lower disease specific quality of life. It was clear that treatment of genital warts is associated with considerable time loss and expenditure to patients.

\section{References}

1 National STD AIDS Control Programme. (online) http://www.aidscontrol.gov.lk accessed 01 January 2017

2 Clinical Effectiveness Group, British Association for Sexual Health and HIV. UK National Guidelines on the Management of Anogenital Warts 2015

3 Badia X, Antonio J, Lara N, Liorens M A, Olmos L, Terros M S L et al Combination of qualitative and quantitative methods for developing a new health related quality of life measure for patient with genital warts.. Health and Quality of Life Outcomes 2005;

4 Official statistics, sexually transmitted infections (STIs) Government UK (online) http://www.gov.uk (accessed 01 January 2017)

5 BuveA, Gourbin C, Laga M. Gender and Sexually transmitted Diseases. King Homes (ed). Sexually Transmitted Diseases, 4th ed. : The McGraw Hill companies; ; 2008. pp. 178 - 192.

6 Aral SO, Holmes KK. The epidemiology of STIs and Their Social and Behavioral Determinants. King Homes (ed). Sexually Transmitted Diseases, 4th ed. : The McGraw Hill companies; ; 2008. pp. 80 - 117.

7 Vener J D. Natural History of genital warts. : ; 1971.

8 . Rachel L. Winer and Laura A. Koutsy. Genital Human Papilloma virus Infection. King Homes (ed). Sexually Transmitted Diseases, 4th ed. : The McGraw Hill companies; ; 2008. pp. 489 - 500.

9 CDC, Sexually transmitted diseases Treatment Guideline.

10 Human Papilloma Virus (HPV) Vaccination Expert Committee Review. Epidemiology Unit (online) https://www.epid.gov.lk (accessed 01 January 2017) 